\title{
Quantum Bose-Fermi droplets
}

Debraj Rakshit ${ }^{1}$, Tomasz Karpiuk ${ }^{2}$, Mirosław Brewczyk ${ }^{2}$, Mariusz Gajda ${ }^{1}$

1 Institute of Physics, Polish Academy of Sciences, Aleja Lotników 32/46, PL-02668 Warsaw, Poland

2 Wydział Fizyki, Uniwersytet w Białymstoku, ul. K. Ciołkowskiego 1L, 15-245 Białystok, Poland

February 20, 2019

\begin{abstract}
We study stability of a zero temperature mixture of attractively interacting degenerate bosons and spin-polarized fermions in the absence of confinement. We demonstrate that higher order corrections to the standard mean field energy of the system can lead to a formation of Bose-Fermi liquid droplets - self-bound incompressible systems in a three-dimensional space. The stability analysis of the homogeneous system is supported by numerical simulations of finite systems by explicit inclusion of surface effects. Our results indicate that Bose-Fermi droplets can be realized experimentally.
\end{abstract}

\section{Contents}

1 Introduction

2 Uniform mixture

3 Finite system analysis - hydrodynamic approach 6

4 Finite system analysis - atomic-orbital approach

5 Conclusions $\quad 13$

\begin{tabular}{ll} 
References & 14 \\
\hline
\end{tabular}

\section{Introduction}

Self-bound systems are quite common in nature. They appear at different scales. Atomic nuclei, Helium droplets, or astronomical objects like white dwarfs or neutron stars are some of the prominent examples. Their stabilization mechanism is due to a subtle balance of attractive forces and repulsive interactions. 
Yet another, not known so far, self-bound systems - extremely dilute quantum liquid droplets of ultracold atoms in a mixture of two Bose-Einstein condensates of different species - have been predicted by D. Petrov [1] on a theoretical ground. Soon after this prediction the quantum droplets were unexpectedly discovered in quite different system - in ultracold ${ }^{164}$ Dy atoms, the atoms possessing the largest dipolar magnetic moment among all atomic species [2]. Further experiments followed [3 5], and soon ${ }^{166} \mathrm{Er}$ droplets, with dipole-dipole interactions as a crucial element, were created [6]. Quite recently another self-bound object droplets in a two-component mixture of ${ }^{39} \mathrm{~K}$ atoms entered the stage [7 7 ]. These ones are a direct realization of scenario suggested by Petrov [1].

The quantum liquid droplets having densities of about $10^{15} \mathrm{~cm}^{-3}$, about eight orders of magnitude less than Helium droplets, are the most dilute droplets ever. They also exist in reduced dimension space for both, the dipolar case [10], as well as the mixture case [11, however dimensional crossover region seems to be easier to access in experiments, [12, 13]. Droplets of ultracold atoms are stabilized against a collapse by quantum fluctuations, i.e. energy of Bogoliubov vacuum [1]. The stabilization mechanism of quantum droplets is universal. The beyond mean-field effects, responsible for quantum fluctuations, can be incorporated into general mean field description by including the so called Lee-Huang-Yang (LHY) term [14 16] into the standard scheme based on the Gross-Pitaevskii equation [17, 18]. This extended Gross-Pitaevskii (eGP) equation supports a self-bound state [19,20]. In addition to the eGP approach, Monte Carlo techniques, allowing for direct treatment of the beyond mean-field effects, are being employed [21,23].

In a quasi $1 \mathrm{D}$ geometry quantum droplets show many similarities to bright solitons. The common feature is that in both systems a quantum spreading is suppressed. Strongly bound bright solitons in Potassium condensate have been studied recently [24]. The solitons produced in ${ }^{39} \mathrm{~K}$ have very large peek density $\sim 5 \times 10^{14} \mathrm{~cm}^{-3}$, and exist, similarly as droplets, at the edge of collapse of the system. In was shown experimentally [8] that in a mixture of two spin states of ${ }^{39} \mathrm{~K}$ a transition from lower density bright solitons to quantum droplets of higher densities has a character of the first order phase transition. In the crossover region both solitons and droplets exist.

This analogy allows to invoke yet another system supporting bright solitons. The effective interactions between bosons can change their character and become attractive in a 1D ultracold mixture of mutually repealing Bose atoms attracted to polarized Fermi atoms. Appearance of bright solitons can be expected then. This scenario was suggested in 25 where ${ }^{40} \mathrm{~K}$ and ${ }^{87} \mathrm{Rb}$ were fermionic and bosonic agents, respectively. The choice is particularly convenient because of a large natural attraction between the two species. Only recently it was verified experimentally that for appropriately chosen attraction between bosons and fermions, the Bose-Fermi mixture is turned into a train of Bose-Fermi solitons [26].

Effective Bose-Bose interactions become attractive only at the edge of stability of a system [27, 28]. In this paper we want to study such Bose-Fermi systems in the unstable region. The main question we want to pose is if quantum fluctuations contributing to the energy of the Bose component and/or higher order beyond mean-field repulsive interactions between Bose and Fermi species can stabilize the mixture and lead to a formation of dilute quantum liquids in the limit of weak interactions. Although our motivation roots in elongated quasi 1D systems, we focus here on the generic 3D case having in mind the ultracold Bose-Fermi mixture of ${ }^{133} \mathrm{Cs}-{ }^{6} \mathrm{Li}$ and recent experiments of Cheng Chin group [29]. 


\section{Uniform mixture}

The mean-field energy $E_{0}$ of a uniform system in a volume $V$, having $N_{B}=n_{B} V$ and $N_{F}=$ $n_{F} V$ bosons and fermions, respectively, can be written in the form:

$$
E_{0} / V=\varepsilon_{0}\left(n_{B}, n_{F}\right)=3 \varepsilon_{F} n_{F} / 5+g_{B} n_{B}^{2} / 2+g_{B F} n_{B} n_{F},
$$

where $n_{B}$ and $n_{F}$ are atomic densities, and the consecutive terms (energy densities) correspond to: (1) a kinetic energy of fermions with $\varepsilon_{F}=\hbar^{2} k_{F}^{2} / 2 m_{F}=5 \kappa_{k} n_{F}^{2 / 3} / 3$ being the Fermi energy, and $k_{F}=\left(6 \pi^{2} n_{F}\right)^{1 / 3}$ the Fermi wave number, (2) a boson-boson interaction energy, and finally (3) a boson-fermion contact interaction energy. For a convenience we introduced the following notation: $\kappa_{k}=(3 / 10)\left(6 \pi^{2}\right)^{2 / 3} \hbar^{2} / m_{F}, g_{B}=4 \pi \hbar^{2} a_{B} / m_{B}$, and $g_{B F}=2 \pi \hbar^{2} a_{B F} / \mu$, where $a_{B}$ $\left(a_{B F}\right)$ is the scattering length corresponding to the boson-boson (boson-fermion) interactions and $m_{B}, m_{F}$, and $\mu=m_{B} m_{F} /\left(m_{B}+m_{F}\right)$ are the bosonic, fermionic, and reduced masses, respectively.

We assume weak interaction limit, i.e. the gas parameters are small: $n_{B}^{1 / 3} a_{B} \ll 1$ and $n_{F}^{1 / 3} a_{B F} \ll 1$ and then, the kinetic energy of fermions, being proportional to $n_{F}^{5 / 3}$, is the largest contribution to the system energy. It favors spreading of fermionic component all the way to infinity. Similar is the effect of repulsive boson-boson interactions, $g_{B}>0$. However, a sufficiently strong attraction, $g_{B F}<0$, can suppress this expansion, but the equilibrium reached is unstable. Higher order terms must come into a play to ensure stability. Perturbative approach suggests the Lee-Huang-Yang term (LHY), the zero-point energy of Bogoliubov vacuum of the Bose system:

$$
E_{L H Y} / V=\varepsilon_{L H Y}\left(n_{B}, n_{F}\right)=C_{L H Y} n_{B}^{5 / 2}
$$

with $C_{L H Y}=64 /(15 \sqrt{\pi}) g_{B} a_{B}^{3 / 2}$. However, this is not enough to stop the system from expansion. Our studies indicate that contribution to the mutual boson-fermion interaction resulting from the higher order term in the Bose-Fermi coupling turns out to be the most important. These effects were considered on a theoretical ground [30 32]. A Bose-Fermi system across a broad Feshbach resonance was studied in [32, but results obtained are applicable only when fermionic density is much larger than density of bosons. As it will be shown later, this situation does not support droplets formation. Contribution to the Bose-Fermi interaction energy obtained in a frame of the second order perturbation theory in [31, more general than results of [30] based on the renormalized T-matrix expansion, leads to the following quantum correction to the energy:

$$
\begin{aligned}
E_{B F} / V & =\varepsilon_{B F}\left(n_{B}, n_{F}\right)=\varepsilon_{F} n_{B}\left(n_{F} a_{B F}^{3}\right)^{2 / 3} A(w, \alpha) \\
& =C_{B F} n_{B} n_{F}^{4 / 3} A(w, \alpha),
\end{aligned}
$$

where $w=m_{B} / m_{F}$ and $\alpha=2 w\left(g_{B} n_{B} / \varepsilon_{F}\right)$ are the dimensionless parameters, $C_{B F}=$ $\left(6 \pi^{2}\right)^{2 / 3} \hbar^{2} a_{B F}^{2} / 2 m_{F}$, and the function $A(w, \alpha)$ is given in a form of integral [31]

$$
\begin{aligned}
& A(w, \alpha)=\frac{2(1+w)}{3 w}\left(\frac{6}{\pi}\right)^{2 / 3} \int_{0}^{\infty} \mathrm{d} k \int_{-1}^{+1} \mathrm{~d} \Omega \\
& {\left[1-\frac{3 k^{2}(1+w)}{\sqrt{k^{2}+\alpha}} \int_{0}^{1} \mathrm{~d} q q^{2} \frac{1-\Theta\left(1-\sqrt{q^{2}+k^{2}+2 k q \Omega}\right)}{\sqrt{k^{2}+\alpha}+w k+2 q w \Omega}\right],}
\end{aligned}
$$


where $\Theta(x)$ is the step theta-function. The arguments of $A(w, \alpha)$ function are: $w=m_{B} / m_{F}$ and $\alpha=2 w\left(g_{B} n_{B} / \varepsilon_{F}\right)=16 \pi n_{B} a_{B}^{3} /\left(6 \pi^{2} n_{F} a_{B}^{3}\right)^{2 / 3}$. The above formula, Eq. (3), for $\alpha \ll 1$, i.e. in the limit when the Fermi energy is much larger than the chemical potential of bosons (assuming the mass ratio, $w$, is of the order of one) coincides with the results of [30].

Summarizing the above discussion, in the regime where both gas parameters are small, $a_{B} n_{B}^{1 / 3} \ll 1$ and $a_{B F} n_{F}^{1 / 3} \ll 1$, we approximate the energy of the dilute uniform system by the following expression:

$$
E\left(N_{B}, N_{F}, V\right)=E_{0}+E_{L H Y}+E_{B F}=V \varepsilon\left(n_{B}, n_{F}\right) .
$$

To find densities corresponding to the energy minimum, physical constrains should be introduced. For a trapped system they are set by number of atoms in every component. Here the system is free, and we look for such a configuration which is stable in absence of any external confinement. Therefore neither the volume $V$, nor the number of atoms $N_{B}$ and $N_{F}$, are controlled. The important role plays a pressure instead, $p\left(n_{B}, n_{F}\right)=-\mathrm{d} E / \mathrm{d} V$. Outside of the droplet it is equal to zero. The same must be inside. We ignore for a moment a surface tension and consider infinite system. For finite droplet, the energy related to the surface is proportional to its area $\propto V^{2 / 3}$, thus is only a fraction $\propto V^{-1 / 3}$ of the internal energy of a droplet. This fraction vanishes in the limit of infinite system, reducing the importance of the surface effects. This fact will be demonstrated in the next section. With all limitations mentioned above, the condition of vanishing pressure is necessary for the mechanical stability of the system:

$$
p\left(n_{B}, n_{F}\right)=n_{B} \mu_{B}+n_{F} \mu_{F}-\varepsilon\left(n_{B}, n_{F}\right)=0,
$$

where we introduced the chemical potentials $\mu_{B(F)}=\mathrm{d} E / \mathrm{d} N_{B(F)}=\partial \varepsilon / \partial n_{B(F)}$ of both species. Numerical solutions of Eq. (6) are shown graphically in Fig. 1 for several values of $a_{B F} / a_{B}$ and in the case of ${ }^{133} \mathrm{Cs}-{ }^{6} \mathrm{Li}(w=22.095)$ mixture (contours for ${ }^{41} \mathrm{~K}-{ }^{40} \mathrm{~K}(w=1.025)$ system look similar). Pressure vanishes at the closed contours forming a kind of loops in $n_{B}-n_{F}$ plane. If $\left|a_{B F}\right| / a_{B}$ is less than some critical value, $\left|a_{B F}\right| / a_{B}<\eta_{0}$, then Eq. (6) has no solutions. Contours marked by solid lines support negative energy solutions. They shrink with increasing $\left|a_{B F}\right| / a_{B}$.

All points on a single contour define mechanically stable droplets for a given values of interaction parameters. Volume of the droplet is not specified, it is a scale parameter, if fixed, allows to determine the number of particles. What is the most important, the energy of the system (for a fixed volume) varies along the contour, and reaches the minimal value, if:

$$
\mu_{B} \frac{\partial p}{\partial n_{F}}-\mu_{F} \frac{\partial p}{\partial n_{B}}=0
$$

Eq. (7) originates in a necessary condition for the extremum of the energy density $\varepsilon\left(n_{B}, n_{F}\right)$ constrained to the zero-pressure line. The energy minima are marked in Fig. 1 by dots. Only these particular spots define systems which are stable with respect to evaporation process. If the initial ratio of number of particles of the Bose and Fermi components is different than corresponding to the above mentioned solution, some particles, mostly the excess ones, are simply evaporated from the droplet.

With our choice of the zero energy, the stable self-bound droplets should be characterized by negative energy. For some range of the parameter, $\eta_{0}<\left|a_{B F}\right| / a_{B}<\eta_{c}$ energies are positive. These are metastable states, marked by broken lines in Fig. 1. Only if $\left|a_{B F}\right| / a_{B}$ exceeds some critical value, $\left|a_{B F}\right| / a_{B}>\eta_{c}$, the energy of droplets becomes negative. These are the stable 


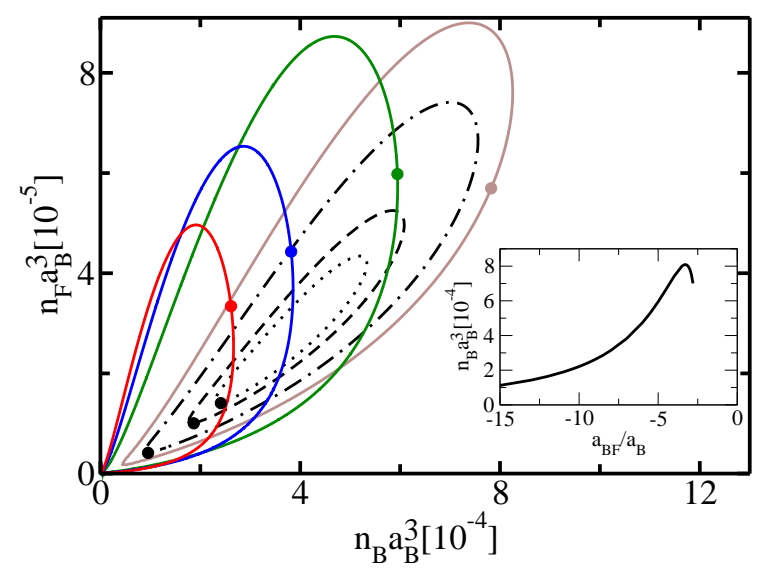

Figure 1: Solutions of Eq. (6), in the form of contour plots in $n_{B}-n_{F}$ plane. ${ }^{133} \mathrm{Cs}-{ }^{6} \mathrm{Li}$ mixture: Broken lines show metastable cases for $a_{B F} / a_{B}=-2.45$ (dotted), -2.5 (dashed), and -2.7 (dash-dotted). Solid lines show stable cases for $a_{B F} / a_{B}=-3$ (brown), -5 (green), -7 (blue), and -9 (red). Dots correspond to the energy minima. The inset shows equilibrium density of the bosonic species as a function of $a_{B F} / a_{B}$ for ${ }^{133} \mathrm{Cs}^{-}{ }^{6} \mathrm{Li}$ mixture.

\begin{tabular}{c|c|c|c|c|c}
\hline & $\eta_{c}$ & $n_{B} a_{B}^{3}$ & $n_{F} a_{B}^{3}$ & $\alpha$ & $m_{B} / m_{F}$ \\
\hline${ }^{41} \mathrm{~K}^{-}{ }^{40} \mathrm{~K}$ & 12.1 & $9.10 \times 10^{-6}$ & $1.26 \times 10^{-6}$ & 0.258 & 1.025 \\
${ }^{87} \mathrm{Rb}{ }^{40} \mathrm{~K}$ & 10.4 & $1.95 \times 10^{-5}$ & $2.01 \times 10^{-6}$ & 0.406 & 2.175 \\
${ }^{133} \mathrm{Cs}^{-6} \mathrm{Li}$ & 2.8 & $7.16 \times 10^{-4}$ & $4.75 \times 10^{-5}$ & 1.796 & 22.095 \\
\hline
\end{tabular}

Table 1: The second column: Values of the critical ratio $\left|a_{B F}\right| / a_{B}$ supporting the existence of stable ${ }^{41} \mathrm{~K}-{ }^{40} \mathrm{~K},{ }^{87} \mathrm{Rb}-{ }^{40} \mathrm{~K}$, and ${ }^{133} \mathrm{Cs}^{-}{ }^{6} \mathrm{Li}$ mixtures. The third and the fourth columns show the corresponding bosonic and fermionic densities. The fourth and fifth ones give the values of $\alpha$ parameter and the mass ratio.

droplets. Values of $\eta_{0}$ and $\eta_{c}$ can be found numerically. In general, except of a small region of the parameter $\left|a_{B F}\right| / a_{B}$, the larger the attraction the smaller the equilibrium densities (see inset of Fig. (1).

In order to reach desired ratio of $\left|a_{B F}\right| / a_{B}$ two approaches are possible. One is to increase the $\left|a_{B F}\right|$, the second is to tune the Bose-Bose scattering length, $a_{B}$, to small values. Both scenarios assume utilizing of appropriate Feshbach resonances. Simultaneously one should avoid large values of densities, as it would lead to a relatively large atom number decay, due to the three-body recombination. This is why a life-time of a droplet is typically of the order of $10 \mathrm{~ms}$ [7,8]. The life-time of Bose-Fermi droplets will be estimated in the following section, where we study dynamical situations.

In the first column of Tab. 1 we list values of the critical ratio $\eta_{c}$ supporting stable liquid droplets, while in the second and third columns we list corresponding densities of Bose and Fermi species. We present the results for three different mixtures of different mass ratio, ${ }^{41} \mathrm{~K}-$ ${ }^{40} \mathrm{~K},{ }^{87} \mathrm{Rb}^{40} \mathrm{~K}$, and ${ }^{133} \mathrm{Cs}^{-}{ }^{6} \mathrm{Li}$. For all these mixtures bosons are in vast majority. Therefore, fermions can be treated as impurity immersed in bosonic cloud bringing analogy to polaron.

Boson-fermion attraction mediates an effective attraction between fermionic atoms. It prevents expansion of fermions due to quantum pressure. Similar mechanism, effective attraction between distinct electrons mediated by interaction with phonons is responsible for a formation 
of Cooper pairs. The question of fermionic superfluidity of Bose-Fermi droplets seems to be legitimate. The same interaction induce an effective attraction between bosons. For a large enough number of bosons this might result in a collapse of bosonic component. Then, fermions start to play an important role. They are able to counteract, due to quantum pressure, the collapse of bosonic cloud - an analogy with white dwarf and neutron stars becomes immediate. Hence, studying of the 'atomic white dwarfs' in the laboratory seems to be possible with Bose-Fermi droplets.

\section{$3 \quad$ Finite system analysis - hydrodynamic approach}

Now we address the properties of finite Bose-Fermi droplets including the surface effects. Evidently, additional energy terms related to the density gradients has to be considered. Therefore, we apply the local density approximation and add the kinetic energy $E_{k}^{B}=\int d \mathbf{r} \varepsilon_{k}^{B}$ with $\varepsilon_{k}^{B}=\left(\hbar^{2} / 2 m_{B}\right)\left(\nabla \sqrt{n_{B}}\right)^{2}$ for bosonic component to Eq. (55). Similarly, for fermions we add the Weizsäcker correction [33] to the kinetic energy, $E_{k, W}^{F}=\int d \mathbf{r} \varepsilon_{k, W}^{F}$, where $\varepsilon_{k, W}^{F}=$ $\xi\left(\hbar^{2} / 8 m_{F}\right)\left(\nabla n_{F}\right)^{2} / n_{F}$, with $\xi=1 / 9$ [34, 35]. We neglect the contribution due to higher order gradient terms. The total energy of a finite Bose-Fermi droplet is then given by $E\left[n_{B}(\mathbf{r}), n_{F}(\mathbf{r})\right]=\int d \mathbf{r}\left(\varepsilon+\varepsilon_{k}^{B}+\varepsilon_{k, W}^{F}\right)$.

The time evolution of the Bose-Fermi system can be conveniently treated within the quantum hydrodynamics [36]. For that both bosonic and fermionic clouds are viewed as fluids characterized by the density and the velocity fields. Since we assume that bosons occupy a single quantum state, their evolution is governed just by the Schrödinger-like equation of motion which includes the mean-field, the LHY, and the boson-fermion interaction terms. Fermions require a special care, however. It has been already discovered many years ago that oscillations of electrons in a many-electron atom can be described by the hydrodynamic equations [37. We follow this proposal and write the hydrodynamic equations for fermions:

$$
\begin{aligned}
& \frac{\partial}{\partial t} n_{F}=-\nabla\left(n_{F} \vec{v}_{F}\right), \\
& m_{F} \frac{\partial}{\partial t} \vec{v}_{F}=-\nabla\left(\frac{\delta T}{\delta n_{F}}+\frac{m_{F}}{2} \vec{v}_{F}^{2}+g_{B F} n_{B}+\frac{\delta E_{B F}}{\delta n_{F}}\right),
\end{aligned}
$$

where $n_{F}(\mathbf{r}, t)$ and $\left.\vec{v}_{F}(\mathbf{r}, t)\right)$ denote the density and velocity fields of fermionic component, respectively. $T$ is the intrinsic kinetic energy of a fermionic gas and is calculated including the lowest order gradient correction [33 35$]$

$$
\frac{\delta T}{\delta n_{F}}=\frac{5}{3} \kappa_{k} n_{F}^{2 / 3}-\xi \frac{\hbar^{2}}{2 m_{F}} \frac{\nabla^{2} \sqrt{n_{F}}}{\sqrt{n_{F}}}
$$

with $\xi=1 / 9$.

The convenient way to further treat Eqs. (8) is to bring them into a form of the Schrödingerlike equation by using the inverse Madelung transformation [38 40]. This is just a mathematical transformation which introduces the single complex function instead of density and velocity fields used in a hydrodynamic description. Both treatments are equivalent provided the velocity field is irrotational (vanishing vorticity). After the inverse Madelung transformation is applied, the equations of motion describing the Bose-Fermi mixture are turned into 


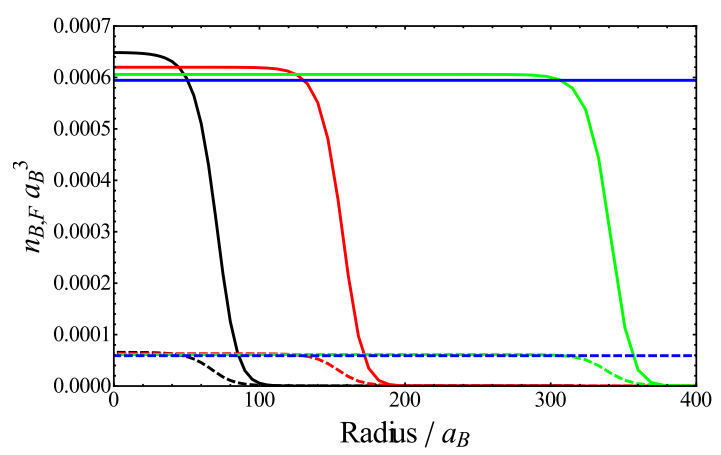

Figure 2: Radial densities (solid and dashed lines for bosons and fermions, respectively) for a sequence of Bose-Fermi droplets. for ${ }^{133} \mathrm{Cs}-{ }^{6} \mathrm{Li}$ mixture for $a_{B F} / a_{B}=-5$ and the initial number of bosons (fermions) equal to 1000 (100), 10000 (1000), and 100000 (10000). The horizontal lines are the bosonic and fermionic densities coming from the analysis ignoring the surface effects. Clearly, the surface effects for larger droplets can be neglected.

coupled Schrödinger-like equations for a condensed Bose field $\psi_{B}\left(n_{B}=\left|\psi_{B}\right|^{2}\right)$ and a pseudowavefunction for fermions $\psi_{F}=\sqrt{n_{F}} \exp (i \phi)\left(n_{F}=\left|\psi_{F}\right|^{2}\right.$ and $\left.\vec{v}_{F}=\left(\hbar / m_{F}\right) \nabla \phi\right)$

$$
\begin{aligned}
i \hbar \frac{\partial \psi_{B}}{\partial t} & =\left[-\frac{\hbar^{2}}{2 m_{B}} \nabla^{2}+g_{B}\left|\psi_{B}\right|^{2}+\frac{5}{2} C_{L H Y}\left|\psi_{B}\right|^{3}\right. \\
& +g_{B F}\left|\psi_{F}\right|^{2}+C_{B F}\left|\psi_{F}\right|^{8 / 3} A(\alpha) \\
& \left.+C_{B F}\left|\psi_{B}\right|^{2}\left|\psi_{F}\right|^{8 / 3} \frac{\partial A}{\partial \alpha} \frac{\partial \alpha}{\partial n_{B}}\right] \psi_{B}, \\
i \hbar \frac{\partial \psi_{F}}{\partial t} & =\left[-\frac{\hbar^{2}}{2 m_{F}} \nabla^{2}+\xi^{\prime} \frac{\hbar^{2}}{2 m_{F}} \frac{\nabla^{2}\left|\psi_{F}\right|}{\left|\psi_{F}\right|}+\frac{5}{3} \kappa_{k}\left|\psi_{F}\right|^{4 / 3}\right. \\
& +g_{B F}\left|\psi_{B}\right|^{2}+\frac{4}{3} C_{B F}\left|\psi_{B}\right|^{2}\left|\psi_{F}\right|^{2 / 3} A(\alpha) \\
& \left.+C_{B F}\left|\psi_{B}\right|^{2}\left|\psi_{F}\right|^{8 / 3} \frac{\partial A}{\partial \alpha} \frac{\partial \alpha}{\partial n_{F}}\right] \psi_{F} .
\end{aligned}
$$

Here, $\xi^{\prime}=1-\xi=8 / 9$. The bosonic wave function and the fermionic pseudo-wave function are normalized as $N_{B, F}=\int d \mathbf{r}\left|\psi_{B, F}\right|^{2}$.

To find the ground state of the Bose-Fermi droplet we solve Eqs. (10) using the imaginary time propagation technique [41. The resulting ground state densities for ${ }^{133} \mathrm{Cs}-{ }^{6} \mathrm{Li}$ mixture for three different numbers of bosons and fermions are shown in Fig. 2, For the smallest droplets the peak densities for both bosons and fermions are slightly higher than predicted by the analysis based on a uniform mixture. For bigger droplets the peak density approaches the uniform mixture solution as expected, because the surface effects become less important. The stability of the droplets is verified by the real time propagation of Eqs. (10). The conclusion is that the droplet reaches the state of the minimal energy by evaporating mostly surplus atoms.

Next, we check whether Bose-Fermi droplets can be formed dynamically in a process of opening a harmonic trap where a mixture of bosonic and fermionic gases is prepared initially. For that we choose the ${ }^{133} \mathrm{Cs}{ }^{-6} \mathrm{Li}$ mixture and set $a_{B}=250 a_{0}$, where $a_{0}$ is the Bohr radius and $a_{B F} / a_{B}=-2.0$ and -3.6 . Initial numbers of atoms are: $N_{B}=100000$, and $N_{F}=10000$. They are confined in the spherically symmetric harmonic traps with frequencies $\omega_{B} /(2 \pi)=$ 




Figure 3: Width of bosonic component of the Bose-Fermi droplet composed of $N_{B}=100000$ bosons and $N_{F}=10000$ fermions as a function of time. The trapping potential is removed in $1 \mathrm{~ms}$ (marked by a vertical line). The solid (dashed) line corresponds to $a_{B F} / a_{B}=-2.0$ $\left(a_{B F} / a_{B}=-3.6\right)$. For the ratio $\left|a_{B F}\right| / a_{B}$ equal to 2.0 , which is below the critical value, both the bosonic and fermionic clouds spread out. In the case when $\left|a_{B F}\right| / a_{B}=3.6$, i.e. larger than the critical one, the breathing droplet is formed. The width of fermionic part of a droplet behaves similarly and is not shown.

$200 \mathrm{~Hz}$ for bosons and $\omega_{F} /(2 \pi)=940 \mathrm{~Hz}$ for fermions. The trap parameters are chosen to match a radius of a droplet to be formed. We find the ground state of such a Bose-Fermi mixture by solving Eqs. (10) in imaginary time. Next, the confinement is removed in $1 \mathrm{~ms}$ $\left(2730 m_{B} a_{B}^{2} / \hbar\right)$. We monitor the evolution of the system by a period of about $8 \mathrm{~ms}$. The width of bosonic component, $\int d \mathbf{r} r\left|\psi_{B}\right|^{2} / N_{B}$, is shown in Fig. 3. Fermionic width looks similar. Vertical line indicates the moment of time when the confinement is completely switched off. The densities preserve the spherical symmetry during the evolution. Clearly, the system stays bound and a droplet is formed when the ratio $\left|a_{B F}\right| / a_{B}$ is above the critical value equal to about 2.8 (see dashed line in Fig. 3). Contrary, for low enough ratio $\left|a_{B F}\right| / a_{B}$ the bosonic and fermionic clouds spread out (solid line in Fig. 3).

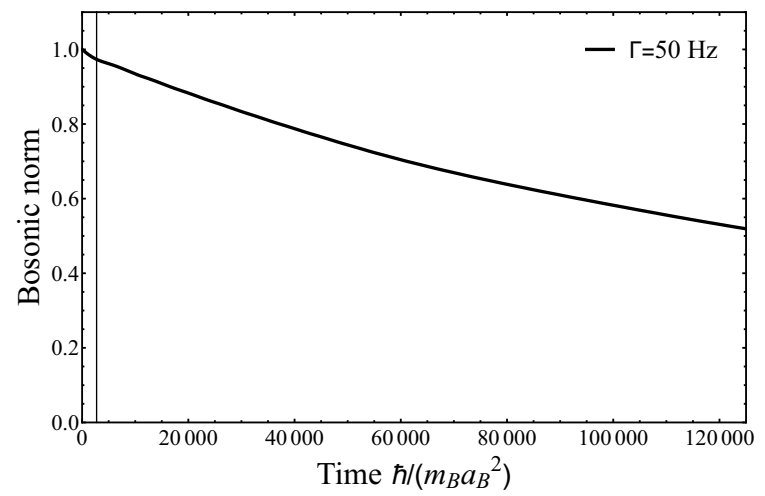

Figure 4: Bosonic norm, with three-body losses included, as a function of time for the ratio $\left|a_{B F}\right| / a_{B}=3.6$ and the numbers of atoms as in Fig. 3, The trapping potential is removed in $1 \mathrm{~ms}$ (marked by a vertical line). The solid black line corresponds to the loss rate equal to $50 / \mathrm{s}$.

Finally, we address the issue of a life-time of a Bose-Fermi droplet due to three-body re- 
combination processes. For that we solve Eqs. (10), with additional terms representing losses resulting from Li-Cs-Cs collisions, included. Therefore, the right-hand side of Eqs. (10) is modified by adding $-i K_{3} / 2 n_{B} n_{F}$ and $-i K_{3} / 2 n_{B}^{2}$ terms for bosonic and fermionic components, respectively. Here, $K_{3}$ is the rate coefficient and its value is estimated by us from the measured losses of Cs atoms (see Fig. 4b in [29]). From the rate equation $\left(1 / N_{B}\right) d N_{B} / d t=-K_{3} n_{B}^{2}$ one finds for $\Gamma=\left(1 / N_{B}\right) d N_{B} / d t=10 / \mathrm{s}$ and the bosonic density $n_{B}=4 \times 10^{14} \mathrm{~cm}^{-3}$, therefore the rate coefficient can be estimated as $K_{3}=6.25 \times 10^{-29} \mathrm{~cm}^{6} / \mathrm{s}$. This rate coefficient scales as $a_{B F}^{4}$. For $a_{B F} / a_{B}=-3.6$ and $a_{B}=250 a_{0}$ one has $\Gamma \approx 50 / \mathrm{s}$ (Fig. $4 \mathrm{~b}$ in [29]). Fig. 4 shows how the norm of bosonic component of the Bose-Fermi droplet (i.e. the number of bosons normalized to its initial value) decreases in time due to three-body losses with the rate $\Gamma \approx 50 / \mathrm{s}$. For $a_{B}=250 a_{0}$ Fig. 4 covers the duration of $45 \mathrm{~ms}$ and hence from Fig. 4 we estimate the life-time of the droplet to be about $45 \mathrm{~ms}$.

\section{Finite system analysis - atomic-orbital approach}

Now we address the properties of finite Bose-Fermi droplets by using the Hartree-Fock approximation in which, as opposed to a hydrodynamic approach, fermions are treated individually. Therefore, we assign a single-particle orbital to each fermionic atom, $\psi_{j}^{F}(\mathbf{r})$, where $j=1, \ldots, N_{F}$ and assume that the many-body wave function of the droplet is a product of the Hartree ansatz for bosons (all bosonic atoms occupy the same state $\psi_{B}$ ) and the Slater determinant, built of orbitals $\psi_{j}^{F}$, for fermions. Hence, the total fermionic and bosonic densities are $n_{F}=\sum_{j}^{N_{F}}\left|\psi_{j}^{F}\right|^{2}$ and $n_{B}=N_{B}\left|\psi_{B}\right|^{2}$, respectively. The easiest way to derive the Hartree-Fock equations of motion for the Bose-Fermi droplet is to extend our analysis performed for a uniform system (see Eq. 5) by applying the local density approximation (as we did previously) and by adding the kinetic energy density terms related to the spatial gradients of fermionic orbitals $E_{k}^{F}=\sum_{j}^{N_{F}} \int d \mathbf{r} \hbar^{2} /\left(2 m_{F}\right)\left(\nabla \psi_{j}^{F *}\right) \nabla \psi_{j}^{F}$, and bosonic wave function $E_{k}^{B}=\int d \mathbf{r} \hbar^{2} /\left(2 m_{B}\right)\left(\nabla \psi_{B}^{*}\right) \nabla \psi_{B}$. The total energy of the droplet, $E+E_{k}^{B}+E_{k}^{F}$, can be now considered as a functional of bosonic wave function, $\psi_{B}(\mathbf{r})$, and fermionic orbitals, $\psi_{j}^{F}(\mathbf{r})$. The time-dependent Hartree-Fock equations are then given by

$$
\begin{aligned}
i \hbar \frac{\partial \psi_{B}}{\partial t} & =\left[-\frac{\hbar^{2}}{2 m_{B}} \nabla^{2}+g_{B} n_{B}+\frac{5}{2} C_{L H Y} n_{B}^{3 / 2}+g_{B F} n_{F}\right. \\
& \left.+C_{B F} n_{F}^{4 / 3} A(\alpha)+C_{B F} n_{B} n_{F}^{4 / 3} \frac{\partial A}{\partial \alpha} \frac{\partial \alpha}{\partial n_{B}}\right] \psi_{B} \\
i \hbar \frac{\partial \psi_{j}^{F}}{\partial t} & =\left[-\frac{\hbar^{2}}{2 m_{F}} \nabla_{j}^{2}+g_{B F} n_{B}+\frac{4}{3} C_{B F} n_{B} n_{F}^{1 / 3} A(\alpha)\right. \\
& \left.+C_{B F} n_{B} n_{F}^{4 / 3} \frac{\partial A}{\partial \alpha} \frac{\partial \alpha}{\partial n_{F}}\right] \psi_{j}^{F}
\end{aligned}
$$

for $j=1, \ldots, N_{F}$.

We solve the Hartree-Fock Eqs. (11) to obtain the densities of a Bose-Fermi droplet consisting of a small number of fermions, see Fig. 5. These results are compared to the results we demonstrated in the previous section, which were achieved within the hydrodynamic description of the Bose-Fermi mixture. The atomic-orbital approach has been used by us previously to study dynamics of Bose-Fermi solitons in quasi-one-dimensional mixtures [25,27] 
(observed experimentally in [26]) as well as fermionic mixtures, in particular in the context of a formation of Cooper pairs [42].
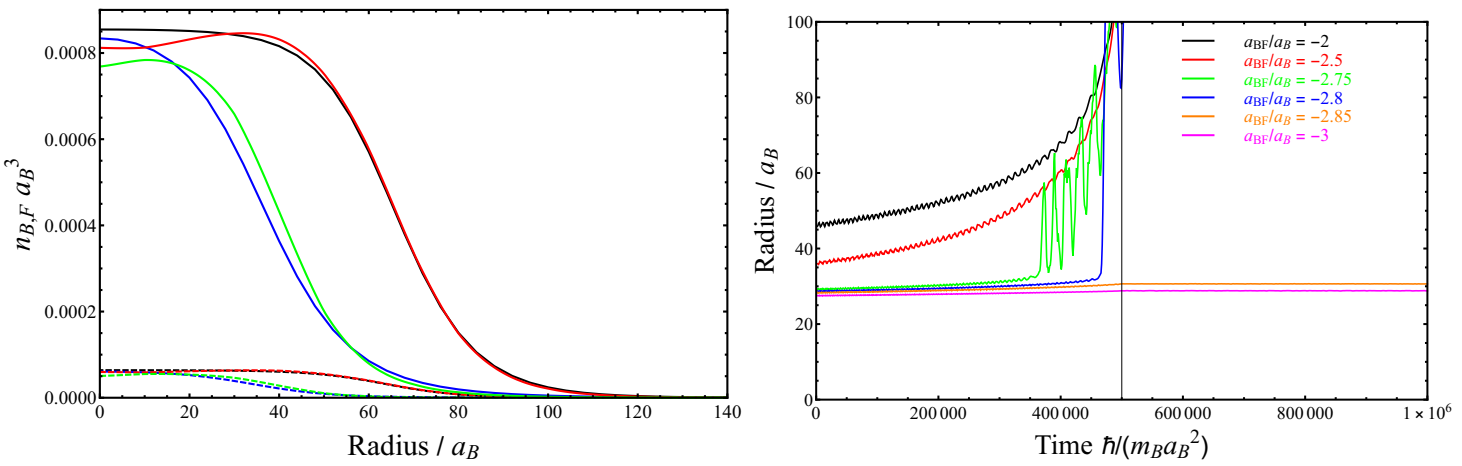

Figure 5: Left frame: Radial densities of bosonic (solid lines) and fermionic (dashed lines) components for two Bose-Fermi droplets. Blue (hydrodynamical approach) and green (atomicorbital method) colors correspond to the droplet consisting of 35 fermions and 350 bosons whereas colors black and red describe the case for 120 fermions and 1250 bosons. In both cases $a_{B F}=-3 a_{B}$. Right frame: Radius of the mixture consisting of 35 fermions and 350 bosons for different values of coupling constants $a_{B F}$, according to the legend, as a function of time. The time is counted from the moment when the trap starts to be removed (at the time marked by a vertical line the trap is fully removed). Both stable $\left(\left|a_{B F}\right| / a_{B}>2.8\right)$, i.e. leading to formation of a droplet and unstable $\left(\left|a_{B F}\right| / a_{B} \leq 2.8\right)$ cases are shown.

Equilibrium densities are plotted in Fig. 5, left frame. The solutions of Eqs. (11) were obtained by adiabatic following of the initially noninteracting trapped systems of $N_{F}$ fermions and $N_{B}$ bosons. Additional trapping terms in Eqs. (11) are included. The traps are chosen to match the size of the droplet to be formed. First, we gradually turned on the mutual interactions between species. In duration of $3 \times 10^{5} m_{B} a_{B}^{2} / \hbar$ the interaction strength is changed from zero to $a_{B F}=-3 a_{B}$. After that the harmonic trap is slowly removed within time interval equal to $5 \times 10^{5} m_{B} a_{B}^{2} / \hbar$. Finally, we monitor the droplet during the further period of $5 \times 10^{5} m_{B} a_{B}^{2} / \hbar$. The system is stable and densities are shown in Fig. [5, left frame. Already for as little as tens of fermions, the atomic-orbital and hydrodynamic descriptions give very similar results. On the other hand, in the right frame in Fig. 5 we demonstrate how the properties of the Bose-Fermi mixture depend on the value of the mutual scattering length $a_{B F}$. Similarly to the hydrodynamic description (see Fig. 3), only for large enough $\left|a_{B F}\right| / a_{B}(>2.8)$ the droplet is formed, otherwise we observe an expansion of both atomic clouds. Note however, that for $\left|a_{B F}\right| / a_{B}$ close to (but below) the critical value, the size of the droplet stays constant for a relatively long time, and then rapidly explodes just before the trap is totally removed. The radius of droplets, on a top of the overall growth, starts to oscillate with growing amplitude when the critical value of $a_{B F}$ is approached. For values of parameters deeper in the unbound regime, the expansion starts rather smoothly just after opening a trap and the amplitude of oscillations of the radius is small, resembling the result obtained by the hydrodynamic approach in the unstable regime, Fig. 3.

The similar outcome of hydrodynamic and Hartree-Fock calculations should not be any surprise. This is because the Madelung transformation, we invoked while solving the hydrodynamic equations in Sec. 3 can be safely used as long as the hydrodynamic velocity field is irrotational. Of course, this is not a general feature of a hydrodynamic flow. For exam- 
ple, when vortices are present in the system, the phase of pseudo-wavefunction is not defined at the positions of vortices cores and the equivalence between the Madelung and hydrodynamic approaches is broken. The other example is related to the interference effect studied for fermionized bosons in the article of Girardeau et al. 43. Here, two interfering clouds of fermions moving irrotationally can not be described as a single fluid with a potential flow. This is because two fermionic clouds are well separated (the fermionic cloud is not simply connected) before they are merged and the total velocity (calculated as a ratio of the total current to the total density) cannot be related to the gradient of the phase in the region of vanishing density. However, in the process of adiabatic formation of the Bose-Fermi droplets the velocity field is irrotational and can be defined everywhere, therefore both hydrodynamic and Hartree-Fock descriptions give similar outcome.

Adiabatic following of the ground state of the Bose-Fermi mixture is a very effective method of finding a stationary droplet as well as occupied single particle orbitals corresponding to the Hartree-Fock Eqs. (11). The stationary version of these equations has a form of the eigenvalue problem. Solving this problem is numerically much more demanding (since it requires the use of large number of basis functions) but gives more insight as the fermionic orbitals and their energies are determined simultaneously. Even more, it gives not only the lowest energy orbitals occupied by a given number of fermionic atoms, but also higher energy single-particle states which could possibly be populated by fermions.
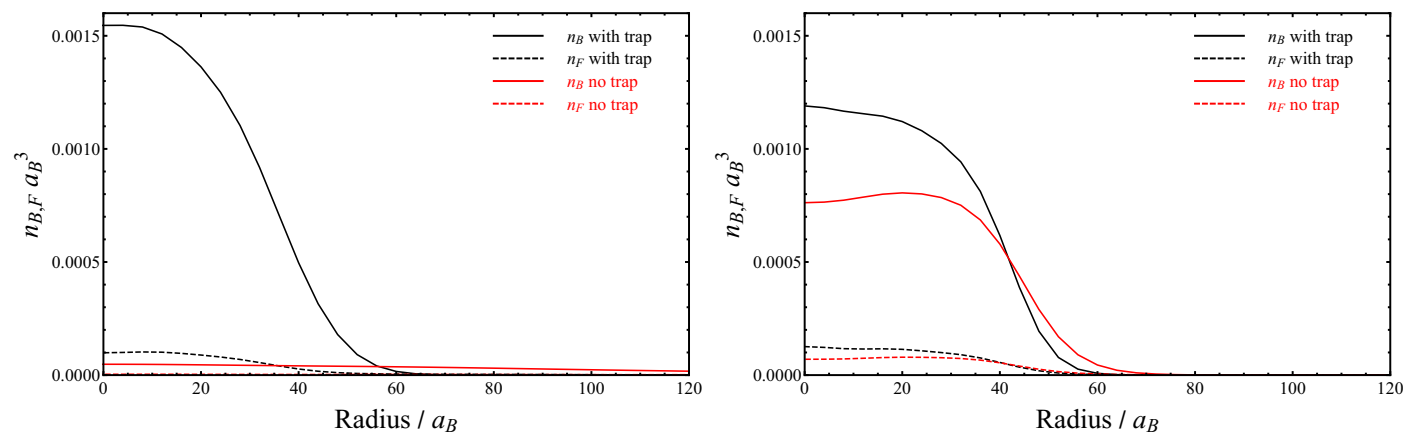

Figure 6: Bosonic densities (solid lines) and fermionic densities (dashed lines) for 35 fermions and 350 bosons - in the harmonic trap (black) and in the free space (red), for the mutual scattering length: $a_{B F} / a_{B}=-2$ (left panel) and $a_{B F} / a_{B}=-4$ (right panel). In the left panel it is clearly visible that if the trap is removed the atoms are uniformly smeared over the entire grid. Fermionic density is so low that it is out of scale and is not visible in figure. Contrary, in the right panel, after the atoms are released from the trap the densities reach equilibrium values corresponding to stationary droplets.

As previously, at the beginning we turn on the harmonic traps and decouple bosons and fermions, i.e. we put $a_{B F}=0$. We find the density of bosonic component by solving the first equation in Eqs. (11) by imaginary time technique. Then we solve the eigenvalue problem for the set of fermions

$$
\begin{aligned}
{\left[-\frac{\hbar^{2}}{2 m_{F}} \nabla_{j}^{2}+\right.} & V_{F T}+g_{B F} n_{B}+\frac{4}{3} C_{B F} n_{B} n_{F}^{1 / 3} A(\alpha) \\
& \left.+C_{B F} n_{B} n_{F}^{4 / 3} \frac{\partial A}{\partial \alpha} \frac{\partial \alpha}{\partial n_{F}}\right] \psi_{j}^{F}=\varepsilon_{j}^{F} \psi_{j}^{F},
\end{aligned}
$$

where $V_{F T}$ represents the harmonic trapping energy for fermions. To this end the harmonic 

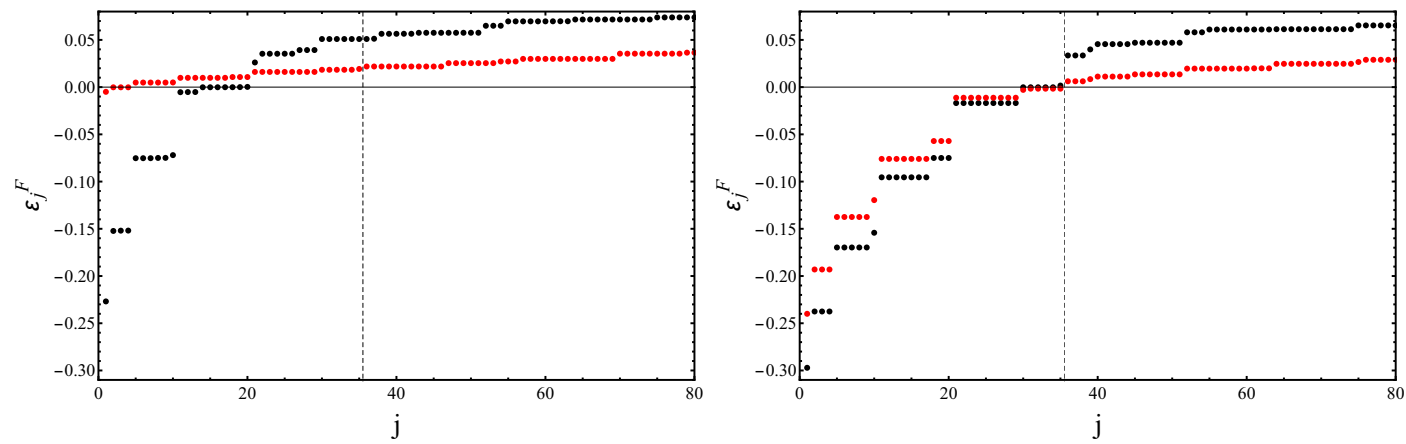

Figure 7: Single-particle energies for the system of 35 fermions immersed in a cloud of 350 bosons - in the harmonic trap (black dots), and without the trap (red dots), for the mutual scattering length equal to: $a_{B F} / a_{B}=-2$ (left panel) and $a_{B F} / a_{B}=-4$ (right panel). The solid horizontal line indicates zero energy level. The dashed vertical line is located at $j=35.5$ which is exactly between 35 th and 36 th fermionic orbital. The total number of basis functions is 2600 .

oscillator wave functions basis is used, and the effective Hamiltonian matrix, as in Eqs. (12), is diagonalized. The eigenvectors define a new fermionic density which, in turn, allows to built next-iteration Hamiltonian matrix. The diagonalization is then repeated and the whole cycle is done again until the fermionic orbitals energies are established with a high enough accuracy. The total energy of the system is checked and provided it stabilizes within assumed accuracy, we move to the next step and change the scattering length $a_{B F}$ by $\Delta a_{B F}=-1 a_{B}$ and the whole procedure is repeated. Otherwise, we do the imaginary time evolution for bosons and iterative procudure for fermions again.

Here, we report results of such an approach for ${ }^{133} \mathrm{Cs}-{ }^{6} \mathrm{Li}$ mixture consisting of $N_{B}=350$ bosons and $N_{F}=35$ fermions. Atoms are confined in the isotropic harmonic trap with frequency $\omega_{B} /(2 \pi)=1200 \mathrm{~Hz}$ for bosons and $\omega_{F} /(2 \pi)=4800 \mathrm{~Hz}$ for fermions. The basis is formed by the 3D harmonic oscillator wave functions. The trap frequency for the basis functions is $\omega /(2 \pi)=12800 \mathrm{~Hz}$. We use this basis functions since they fit better the final size of the droplets.

When the final value of $a_{B F}$ is reached we start to open the trap. We lower the trap strength by $20 \%$ in each of five steps. In Fig. 6 we show the density of bosons and fermions for two qualitatively different attractive scattering lengths. In the left panel $\left|a_{B F}\right| / a_{B}=2$, i.e. is well below the critical value 2.8. When the trap is present (black curves) both components are hold by the trap. When the trap is off then both bosons and fermions spread over all available space (red lines). The red dashed line which indicates the fermionic density is so low that it is not visible in the figure. Contrary, in the right panel of Fig. 6 when the mutual scattering length $\left|a_{B F}\right| / a_{B}=4$ is well above the critical value 2.8 , behavior of the mixture is qualitatively different. Now, when the trap is off, the stable self-bound Bose-Fermi droplet is formed.

This observation is supported by analysis of single-particle energies of fermionic component. These are shown in Fig. 7. The left panel corresponds to the case when $\left|a_{B F}\right| / a_{B}=2$. If the system is trapped some number of single-particle energies are negative. But when the trap is off almost all become positive and form a continuum of above zero energy. This is the case of free particles. The opposite case is when $\left|a_{B F}\right| / a_{B}=4$, i.e. above the critical value 2.8. Now 
all 35 fermions have negative energies as one expects for trapped particles. This is indicated by the vertical dashed line located at 35.5, exactly between 35 th and 36 th single-particle state. There is 8 energy levels below zero energy. One can easily recognize two families of states, corresponding to the radial quantum number equal to 0 and to 1 . Because of the threedimensional spherical geometry the eigenenergies are degenerate, and these eight energy levels are able to accommodate many more fermions than in one-dimension, where only one spinpolarized fermion per one energy level is allowed. Therefore, in three-dimensional Bose-Fermi droplets the number of fermions can be large as opposed to the case of quasi-one-dimensional Bose-Fermi mixtures of magnetic atoms [45].

\section{Conclusions}

Presented here analysis of stability of a mixture of ultracold Bose-Fermi atoms indicate that stable liquid self-bound droplets can be spontaneously formed when interspecies attraction is appropriately tuned. Droplets are stabilized by the higher order term in the Bose-Fermi coupling. We predict the values of interaction strengths as well as atomic densities corresponding to droplets of three different mixtures, suitable for experimental realization, ${ }^{41} \mathrm{~K}-{ }^{40} \mathrm{~K},{ }^{87} \mathrm{Rb}-$ ${ }^{40} \mathrm{~K}$, and ${ }^{133} \mathrm{Cs}^{6}{ }^{6} \mathrm{Li}$. The LHY quantum correction for bosons, which is positive, makes bosonic and fermionic densities low enough to decrease the impact of three-body losses. We demonstrate by time dependent calculations that a Bose-Fermi droplet should be achievable by preparing the mixture of bosonic and fermionic atoms in a trap and then by slowly removing the confinement.

Quantum Bose-Fermi droplets bring into a play the higher order term in Bose-Fermi coupling. The role of this term was not studied extensively in experiments so far. Moreover the effect of this 'correction' seems to be somewhat elusive as reported in [44]. We think that this fact should not discourage future experiments towards investigation of this higher order effects in Bose-Fermi systems. Liquid droplets such as studied here, seem to be the best systems to this end.

The ultradilute self-bound Bose-Fermi droplets are novel, not known before form of the matter organization. Their composition, involving not only bosonic, but also fermionic component, might bring into play a rich variety of physical phenomena related to polaron physics, Cooper pairing mediated by bosons, as well as to fermionic superfluidity. Stabilization mechanism involving Fermi pressure brings some analogies to astronomical objects like white dwarfs or neutron stars. Dynamics of droplets' collisions, their merging and collisions can simulate some astronomical processes as well.

\section{Acknowledgements}

The authors thank Letticia Tarruell for discussions. MG and DR acknowledge support from the EU Horizon 2020-FET QUIC 641122. MG, TK and MB from the (Polish) National Science Center Grant No. 2017/25/B/ST2/01943. Some part of the results were obtained using computers at the Computer Center of University of Białystok. 


\section{References}

[1] D. S. Petrov, Quantum Mechanical Stabilization of a Collapsing Bose-Bose Mixture, Phys. Rev. Lett. 115, 155302 (2015), doi:10.1103/PhysRevLett.115.155302.

[2] H. Kadau, M. Schmitt, M. Wenzel, C. Wink, T. Maier, I. Ferrier-Barbut, and T. Pfau, Observing the Rosensweig instability of a quantum ferrofluid, Nature 530, 194 (2016), doi:/10.1038/nature16485.

[3] I. Ferrier-Barbut, H. Kadau, M. Schmitt, M. Wenzel, and T. Pfau, Observation of quantum droplets in a strongly dipolar Bose gas, Phys. Rev. Lett. 116, 215301 (2016), doi:10.1103/PhysRevLett.116.215301.

[4] I. Ferrier-Barbut, M. Schmitt, M. Wenzel, H. Kadau, and T. Pfau, Liquid quantum droplets of ultracold magnetic atoms, J. Phys. B 49, 214004 (2016), doi: $10.1088 / 0953-4075 / 49 / 21 / 214004$.

[5] M. Schmitt, M. Wenzel, B. Böttcher, I. Ferrier-Barbut, and T. Pfau, Self-bound droplets of a dilute magnetic quantum liquid, Nature 539, 259 (2016), doi:10.1038/nature20126.

[6] L. Chomaz, S. Baier, D. Petter, M. J. Mark, F. Wächtler, L. Santos, and F. Ferlaino, Quantum-Fluctuation-Driven Crossover from a Dilute Bose-Einstein Condensate to a Macrodroplet in a Dipolar Quantum Fluid, Phys. Rev. X 6, 041039 (2016), doi:10.1103/PhysRevX.6.041039.

[7] C. R. Cabrera, L. Tanzi, J. Sanz, B. Naylor, P. Thomas, P. Cheiney, and L. Tarruell, Quantum Liquid Droplets in a mixture of Bose Einstein condensates, Science, 359301 (2018) doi:10.1126/science.aao5686.

[8] P. Cheiney, C. R. Cabrera, J. Sanz, B. Naylor, L. Tanzi, L. Tarruell, Phys. Rev. Lett 120 135301, (2018), doi:10.1103/PhysRevLett.120.135301.

[9] G. Semeghini, G. Ferioli, L. Masi, C. Mazzinghi, L. Wolswijk, F. Minardi, M. Modugno, G. Modugno, M. Inguscio, M. Fattori, Phys. Rev. Lett. 120235301 (2018), doi $10.1103 /$ PhysRevLett.120.235301.

[10] C. Mishra, D. Edler, F. Wächtler, R. Nath, S. Sinha, and L. Santos, Quantum droplets in one-dimensional dipolar Bose-Einstein condensates, Phys. Rev. Lett. 119, 050403 (2017), doi:10.1103/PhysRevLett.119.050403.

[11] D. S. Petrov and G. Astrakharchik, Ultradilute low-dimensional liquids, Phys. Rev. Lett. 117, 100401 (2016), doi:10.1103/PhysRevLett.117.100401.

[12] P. Zin, M. Pylak, T. Wasak, M. Gajda, and Z. Idziaszek, Quantum BoseBose droplets at a dimensional crossover, Phys. Rev. A 98, 051603(R) (2018), doi: $10.1103 /$ PhysRevA.98.051603.

[13] T. Ilg, J. Kumlin, L. Santos, D. S. Petrov, and H. P. B”uchler, Dimensional crossover for the beyond-mean-field correction in Bose gases, Phys. Rev. A 98, 051604(R) (2018), doi:10.1103/PhysRevA.98.051604. 
[14] T. D. Lee, K. Huang, and C. N. Yang, Eigenvalues and eigenfunctions of a Bose system of hard spheres and its low-temperature properties. Phys. Rev. 106, 1135 (1957), doi:10.1103/PhysRev.106.1135.

[15] R. Schützhold, M. Uhlmann, Y. Xu, U. R. Fischer, Int. J. Mod. Phys. B 20, 3555 (2006), doi:10.1142/S0217979206035631.

[16] A. R. P. Lima and A. Pelster, Quantum fluctuations in dipolar Bose gases, Phys. Rev. A 84, 041604 (2011), doi:10.1103/PhysRevA.84.041604.

[17] F. Wächtler and L. Santos, Quantum filaments in dipolar Bose-Einstein condensates, Phys. Rev. A 93, 061603(R) (2016), doi:10.1103/PhysRevA.93.061603.

[18] R. N. Bisset, R. M. Wilson, D. Baillie, and P. B. Blakie, Ground-state phase diagram of a dipolar condensate with quantum fluctuations, Phys. Rev. A 94, 033619 (2016), doi:10.1103/PhysRevA.94.033619.

[19] F. Wächtler and L. Santos, Ground-state properties and elementary excitations of quantum droplets in dipolar Bose-Einstein condensates, Phys. Rev. A 94, 043618 (2016), doi:10.1103/PhysRevA.94.043618.

[20] D. Baillie, R. M. Wilson, R .N. Bisset, and P. B. Blakie, Self-bound dipolar droplet: A localized matter wave in free space, Phys. Rev. A 94, 021602(R) (2016), doi:10.1103/PhysRevA.94.021602.

[21] H. Saito, Path-Integral Monte Carlo Study on a Droplet of a Dipolar Bose-Einstein Condensate Stabilized by Quantum Fluctuation, J. Phys. Soc. Jpn. 85, 053001 (2016), doi:10.7566/JPSJ.85.053001.

[22] A. Macia, J. Sánchez-Baena, J. Boronat, F. Mazzanti, Droplets of trapped quantum dipolar bosons, Phys. Rev. Lett. 117, 205301 (2016), doi:10.1103/PhysRevLett.117.205301.

[23] F. Cinti, A. Capellaro, L. Salasnich and T. Macri, Superfluid filaments of dipolar bosons in free space, Phys. Rev. Lett. bf 119, 215302 (2017), doi:10.1103/PhysRevLett.119.215302.

[24] S. Lepoutre, L. Fouché, A. Boissé, G. Berthet, G. Salomon, A. Aspect, and T. Bourdel, Production of strongly bound 39K bright solitons, Phys. Rev A 94, 053626 (2016), doi:10.1103/PhysRevA.94.053626.

[25] T. Karpiuk, M. Brewczyk, S. Ospelkaus-Schwarzer, K. Bongs, M. Gajda, and K. Rzążewski, Soliton trains in Bose-Fermi mixtures, Phys. Rev. Lett. 93, 100401 (2004), doi:10.1103/PhysRevLett.93.100401.

[26] B. J. DeSalvo, K. Patel, G. Cai, and C. Chin, Fermion-Mediated Interactions Between Bosonic Atoms, arXiv:1808.07856.

[27] T. Karpiuk, M. Brewczyk, and K. Rzążewski, Bright solitons in Bose-Fermi mixtures, Phys. Rev. A 73, 053602 (2006), doi:10.1103/PhysRevA.73.053602.

[28] T. Karpiuk, M. Brewczyk, M. Gajda, and K. Rzążewski, On the stability of Bose-Fermi mixtures, J. Phys. B: At. Mol. And Optical Phys. 38, L215 (2005), doi: $10.1088 / 0953-4075 / 38 / 13 /$ L02 . 
[29] B.J. DeSalvo, K. Patel, J. Johansen, and C. Chin, Observation of a degenerate Fermi gas trapped by a Bose-Einstein condensate, Phys. Rev. Lett. 119, 233401 (2017), doi:10.1103/PhysRevLett.119.233401.

[30] A.P. Albus, S.A. Gardiner, F. Illuminati, and M. Wilkens, Quantum field theory of dilute homogeneous Bose-Fermi mixtures at zero temperature: General formalism and beyond mean-field corrections, Phys. Rev. A 65, 053607 (2002) doi:10.1103/PhysRevA.65.053607.

[31] L. Viverit and S. Giorgini, Ground-state properties of a dilute Bose-Fermi mixture, Phys. Rev. A 66, 063604 (2002), doi:10.1103/PhysRevA.66.063604.

[32] Z.-Q. Yu, S. Zhang, and H. Zhai, Stability condition of a strongly interacting bosonfermion mixture across an interspecies Feshbach resonance, Phys. Rev. A 83, 041603(R) (2011), doi:10.1103/PhysRevA.83.041603.

[33] C. F. Weizsäcker, Zur Theorie der Kernmassen, Z. Phys. 96, 431 (1935) doi: $10.1007 / \mathrm{BF} 01337700$

[34] D. A. Kirznits, Sov. Phys. JETP 5, 64 (1957).

[35] G. L. Oliver and J. P. Perdew, Spin-density gradient expansion for the kinetic energy, Phys. Rev. A 20, 397 (1979), doi:10.1103/PhysRevA.20.397.

[36] E. Madelung, Quantentheorie in hydrodynamischer Form, Z. Phys. 40, 322 (1927), doi: $10.1007 / \mathrm{BF} 01400372$.

[37] J. A. Ball, J. A. Wheeler, and E. L. Fireman, Photoabsorption and charge oscillation of the Thomas-Fermi atom, Rev. Mod. Phys. 45, 333 (1973), doi 10.1103/RevModPhys.45.333.

[38] B. Kr. Dey and B. M. Deb, Femtosecond quantum fluid dynamics of helium atom under an intense laser field, Int. J. Quantum Chem. 70, 441 (1998), doi:10.1002/(SICI)1097-461X(1998)70:3<441::AID-QUA3>3.0.CO;2-U,

[39] A. Domps, P.-G. Reinhard, and E. Suraud, Time-dependent Thomas-Fermi approach for electron dynamics in metal clusters, Phys. Rev. Lett. 80, 5520 (1998), doi $10.1103 /$ PhysRevLett.80.5520.

[40] P.T. Grochowski, T. Karpiuk, M. Brewczyk, and K. Rzążewski, Unified description of dynamics of a repulsive two-component Fermi gas, Phys. Rev. Lett. 119, 215303 (2017), doi: $10.1103 /$ PhysRevLett.119.215303.

[41] K. Gawryluk, T. Karpiuk, M. Gajda, K. Rzążewski, and M. Brewczyk, Unified way for computing dynamics of Bose-Einstein condensates and degenerate Fermi gases, Int. J. Comput. Math, 95, 2143 (2018), doi:10.1080/00207160.2017.1370545.

[42] T. Karpiuk, M. Brewczyk, and K. Rzążewski, Ground state of two-component degenerate fermionic gases, Phys. Rev. A 69, 043603 (2004), doi:10.1103/PhysRevA.69.043603.

[43] M.D. Girardeau and E.M. Wright, Breakdown of Time-dependent mean-field theory for a one-dimensional condensate of impenetrable bosons, Phys. Rev. Lett. 84, 5239 (2000), doi:10.1103/PhysRevLett.84.5239. 
[44] Y.-il Shin, A. Schirotzek, Ch. H. Schunck, and W. Ketterle, Realization of a strongly interacting Bose-Fermi mixture from a two-component Fermi gas, Phys. Rev. Lett. 101, 070404 (2008), doi:10.1103/PhysRevLett.101.070404.

[45] M. Wenzel, T. Pfau, and I. Ferrier-Barbut, Physica Scripta 93, 104004 (2018), doi: $10.1088 / 1402-4896 / \operatorname{aadd} 72$. 